\title{
Über die Trommersche Probe beim Harn.
}

\author{
Von
}

E. Salkowski.

(A us der chemischen Abteilung des Pathologischen Instituts der Universität Berlin.) (Der Redaktion zugegangen am 7. Mai 1912.)

Wiederholt bin ich befragt worden, woran es liege, daß ein Harn, der auf Zucker untersucht wurde, reichlich Kupferoxydhydrat mit blauer Farbe löste, beim Erhitzen nur wenig Kupferoxydulhydrat ausschied, oder selbst garnichts, jedenfalls nicht entsprechend seinem auf Traubenzuckergehalt bezogenen Lösungsvermögen für Kupferoxydhydrat. Bei der gemeinsamen Untersuchung, bei der ich ein abnormes Lösungsvermögen nicht konstatieren konnte, stellte sich dann meistens heraus, da $B$ der Untersuchende die Probe anders angestellt hatte, als üblich, nämlich zu dem Harn nicht zuerst Natronlauge hinzugesetzt hatte, dann Kupfersulfatlösung, sondern umgekehrt zuerst Kupfersulfat und dann Natronlauge.

Auf diese größere Löslichkeit des Kupferhydroxyds unter den angegebenen Verhältnissen und die daraus weiterhin sich ergebenden Konsequenzen hat neuerdings Fr. N. Schulz ${ }^{1}$ ) aufmerksam gemacht und dies veranlaßt mich zu einigen Bemerkungen über den Gegenstand.

Die Tatsache selbst ist entsprechend den Angaben von Schulz an jedem einigermaßen konzentrierten Harn - also etwa von 1017-1018 D oder mehr - leicht zu konstatieren:

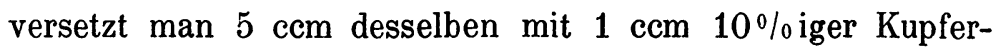
sulfatlösung (bezogen auf $\mathrm{CuSO}_{4}+5 \mathrm{H}_{2} \mathrm{O}$ ) und dem halben Volumen $15 \%$ iger Natronlauge (bezogen auf $100 \mathrm{~g}$, also Natronlauge des D. A. B. 5), so erhält man eine tiefblaue Lösung, während bei dem umgekehrten Verfahren ein großer Teil des Kupferoxydhydrats ungelöst bleibt. Auch wenn man nicht, wie

1) Diese Zeitschrift, Bd. 77, S. 121 (1912). 
ziemlich allgemein üblich, Natronlauge in der angegebenen Quantität hinzusetzt, sondern nur, wie Schulz es tut, 15-20 Tropfen $30 \%$ iger Natronlauge, ist das Verhalten dasselbe.

Bezüglich der Ursache dieser auffallenden Erscheinung meint Schulz, daß dies Lösungsvermögen zu einem großen Teil auf Kreatinin beruhe, da Lösungen desselben beträchtliche Quantitäten von Kupferhydroxyd in Lösung halten könnten, wenn man sie zuerst mit Kupfersulfatlösung, dann mit Natronlauge versetzt, nicht aber umgekehrt. Diese Angabe kann ich durchaus bestätigen: in der Tat ist der Unterschied ein ganz eklatanter, wenn ich mich auch nicht davon überzeugen konnte, daß das Kreatinin gar kein Kupferoxydhydrat löst, wenn man zuerst Natronlauge und dann Kupfersulfat hinzusetzt. Es ist vielleicht nicht überflüssig, wenn ich bemerke, daß ich, wie Schulz, eine ungefähr 1\% ige Kreatininlösung angewendet habe (erhalten durch dreimaliges Verdampfen von Kreatin mit dem 10 fachen an Salzsäure von 1,125 auf dem Wasserbad zur Trockne oder fast zur Trockne). Auf $5 \mathrm{ccm}$ der Kreatininlösung wandte ich 8 Tropfen 10\% iger Kupfersulfatlösung und 1/2 Volumen $30 \%$ iger Natronlauge an. Die tiefblaue Lösung gibt auch eine weit schönere Reduktion beim Erhitzen, wie die in "normaler» Weise hergestellte.

Schulz ist der Ansicht, daß die im Harn vorhandene Quantität Kreatinin zu gering sei, um die fragliche Erscheinung zu erklären, und daß noch andere Stoffe in Betracht kommen müßten und das müßten solche sein, die im Harn in beträchtlicher Quantität vorkommen. Ob Schulz damit recht hat und nicht doch das Kreatinin allein ausreicht, scheint mir etwas zweifelhaft.

Schulz hat nun weiterhin angegeben, daß beim Erhitzen einer so "umgekehrt» angestellten Mischung unter Umständen auch bei normalem Harn eine reichliche Ausscheidung von gelbem oder orangefarbenem Kupferoxydulhydrat eintritt, welche in Verbindung mit dem vorher festgestellten starken Lösungsvermögen für Kupferoxydhydrat leicht zu fälschlicher Annahme von Zuckergehalt führen könne. Diese Ausscheidung von Kupferoxydulhydrat tritt allerdings nicht während des Erhitzens, auch nicht unmittelbar darauf ein, sondern erst nach einigem Zuwarten, 
eventuell auch erst bei nochmaligem Erhitzen. Ein schwach zuckerhaltiger Harn verhält sich aber auch nicht anders oder nicht viel anders. Schulz hat also ganz recht, wenn er die Möglichkeit eines Irrtums für sehr naheliegend hält.

Nun fragt sich aber, ob sie geringer ist, wenn man die Reaktion in der gewöhnlichen, sagen wir «normalen» Weise anstellt. Darunter verstehe ich, daß man den Harn mit dem halben Volumen Natronlauge von ca. 15\% versetzt und dann unter Umschütteln mit soviel Kupfersulfatlösung, als sich irgend «unterbringen» läßt, d. h. so, daß ein Teil des Kupferhydroxyds ungelöst bleibt. ${ }^{1}$ ) Das ist nicht der Fall: die Gefahr besteht, auch wenn man «normal» verfährt. Schon vor mehr als 30 Jahren habe ich mich anheischig gemacht, jeden normalen, nicht $\mathrm{zu}$ dünnen Harn als zuckerhaltig zu demonstrieren, und das ist mir bei dem angegebenen Verfahren des möglichst großen Kupferzusatzes auch wohl ausnahmslos geglückt, $d$. h. wenn man als beweisend für Zucker noch ansieht, daß die Ausscheidung des Oxydulhydrats nach einigem Zuwarten, also vielleicht nach einer halben bis einer Minute eintritt. Man könnte ja die Forderung aufstellen, daß die Ausscheidung von Oxydul, wenn sie für Zuckergehalt beweisend sein soll, schon während des Erhitzens eintreten müsse. Sieht man aber dies als Kriterium für Zucker- . gehalt an, so wird man oft genug einen geringen Zuckergehalt übersehen: ein normaler Harn, in dem man für $100 \mathrm{ccm} \mathrm{0,2,}$ ja selbst $0,4 \mathrm{~g}$ Traubenzucker aufgelöst hat, gibt auch nicht gleich Ausscheidung von Oxydul beim Erhitzen. So wird es denn schließlich Sache der persönlichen Erfahrung, ich möchte beinahe sagen, der Empfindung - , ob man eine Ausscheidung von Oxydul, nach dem Zeitintervall zwischen Erhitzen und Ausscheidung beurteilt, noch als beweisend für Zuckergehalt ansehen soll oder nicht, d. h. das subjektive Moment wird so groß, daß man die Trommersche Probe - einen geringen Zuckergehalt vorausgesetzt - als sehr unsicher ansehen muß.

1) In der Literatur findet sich vielfach die Angabe, bei diesem Verfahren bestehe die Gefahr, daß sich beim Erhitzen schwarzes Kupferoxyd ausscheidet. Das muß ich entschieden bestreiten, man müßte denn Kupfersulfat weit über das Lösungsvermögen hinaus hinzusetzen. 
Nach dem Erscheinen der Schulzschen Arbeit habe ich versucht, ob die Verhältnisse vielleicht günstiger liegen, wenn man nicht das halbe Volumen 15\% iger Natronlauge anwendet, sondern bei normaler Reihenfolge der Reagenzien nur 15 bis 20 Tropfen $30 \%$ iger Natronlauge, wie Schulz angibt. Das ist aber nicht der Fall: der geringere Alkaligehalt ändert nichts an dem Verhalten des normalen und des schwach zuckerhaltigen Harns.

Nun könnte man ja auf die Forderung der Ausscheidung von Oxydul ganz verzichten und sein Urteil auf die Intensität der Reduktion, d. h. der dadurch bewirkten Farbenänderung basieren. Das Gelöstbleiben des Oxyduls kann man bekanntlich erreichen, wenn man nicht möglichst viel Kupfersulfat hinzusetzt, sondern nur soviel, daß man eine blaue Lösung erhält. In der Tat ist es so oft möglich, eine normale Reduktion von einer durch Zuckergehalt bedingten $\mathrm{zu}$ unterscheiden, namentlich wenn man noch die Reaktion auf Kreatinin zu Hilfe zieht, d. h. eine stärkere Reduktion - Auftreten von Gelbfärbung statt der normalen Grün- oder Grüngelbfärbung - dann nicht für beweisend hält, wenn die Reaktion auf Kreatinin, die ich stets neben der Zuckerprobe anstelle, stark ausfällt, für eine allgemeine Anwendung ist dieses Verfahren aber ebensowenig empfehlenswert, wie die Verwendung der Fehlingschen Lösung.

Ich kehre nun noch einmal zu der Frage der Reihenfolge der Reagenzien zurück.

Der Autor der *umgekehrten» Probe ist mir nicht bekannt, in der physiologisch-chemischen Literatur ist sie meines Wissens nicht erwähnt, Schulz war sie auch neu, ich kenne sie jedenfalls nur von Hörensagen; sie soll danach die in der Naunynschen Schule übliche Probe sein. Nun läßt sich a priori annehmen, daß irgend welche Gründe doch zum Gebrauch der «umgekehrten» Probe geführt haben müssen. Ich glaube, daß sich in der Tat einiges zugunsten der «umgekehrten" Probe anführen läßt. In erster Linie der Vorzug größerer Bequemlichkeit und der Zeitersparnis. Es ist nicht jedermanns Sache, den nötigen Kupferzusatz auszuprobieren, erfordert auch viel mehr Zeit, die nicht immer zur Verfügung steht, wenn eine größere 
Anzahl von Harnen zunächst auf Zucker vorgeprüft werden soll. Es kann ja wohl vorkommen, daß $1 \mathrm{ccm}$ einer $10 \%$ igen Kupfersulfatlösung auf $5 \mathrm{ccm}$ Harn zu viel ist, dann wird man zu dem anderen Verfahren zurückkehren müssen. Außerdem habe ich aber auch den Eindruck gehabt, daß man einen normalen Harn, in dem man $0,2 \%$ Traubenzucker aufgelöst hat, durch die umgekehrte Probe leichter von dem normalen Kontrollharn unterscheiden kann, als durch die gewöhnlich angestellte.

Schulz verwirft die «umgekehrte» Probe vollständig als irreführend, darin kann ich mich ihm nicht ganz anschließen, man muß nur immer dessen eingedenk sein, daß die zuerst entstehende blaue Lösung nichts für etwaigen Zuckergehalt bedeutet. Tut man das, so ist die umgekehrte Probe recht wohl brauchbar. Bei Anstellung derselben empfehle ich, die Ver-

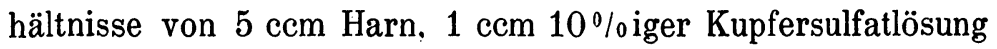
und ungefähr das halbe Volumen 15\% iger Natronlauge innezuhalten.

Man hat sich nun bekanntlich vielfach bemüht, die Trommersche Probe, der doch immer, wie allgemein angenommen wird, eine nicht unerhebliche, nur durch lange Erfahrung etwas abzuschwächende Unsicherheit anhaftet, wenigstens wenn es sich um geringe Zuckermengen handelt, $\mathrm{zu}$ verbessern. Es würde zu weit führen, auf alle in dieser Hinsicht gemachten Vorschläge einzugehen, ich möchte nur auf zwei Modifikationen aufmerksam machen, die mir Vorzüge zu haben scheinen.

1. Wenn man zu $5 \mathrm{ccm} 30 \%$ iger Natronlauge 8 Tropfen $10 \%$ iger Kupfersulfatlösung (mit der Pipette) hinzusetzt und gut durchschüttelt, so erhält man eine klare blaue Lösung, die auch beim Erhitzen zum Sieden sich nicht ändert. Mitunter treten einige schwarze Flöckchen von Kupferoxyd auf, die für die Anstellung der Reaktion ohne Bedeutung sind. Bringt man zu der heißen Lösung $2 \mathrm{ccm}$ Harn und erhitzt nochmals zum Sieden, so ist die Reduktion bei einem 0,2\% Zucker enthaltenden Harn meistens deutlich stärker als beim Kontrollharn. Sehr viel deutlicher wird aber der Unterschied, wenn man 
nach einem schon von Hoppe-Seyler gemachten Vorschlag zu der noch heißen Flüssigkeit vorsichtig an der Wand des Glases Salzsäure zufließen läßt. In der zuckerhaltigen Probe entsteht dann eine beim Schütteln sich zunächst verbreiternde Zone von eigelbem Kupferoxydulhydrat, in der nicht zuckerhaltigen ist diese Erscheinung nur ganz unbedeutend oder sie fehlt ganz. Auch wenn man allmählich unter Durchmischung mit Salzsäure nahezu neutralisiert, besteht ein unzweifelhafter Unterschied in der Farbe und in der Ausscheidung von gelbem Kupferoxydulhydrat zwischen der zuckerhaltigen und der zuckerfreien Probe. Das mag vielleicht nicht ausnahmlos der Fall sein, die Regel ist es jedenfalls.

2. Patéin und Dufau haben bekanntlich zur Klärung des Harns zum Zwecke der Polarisation empfohlen, den Harn mit Quecksilbernitrat zu fällen. Porcher ${ }^{1}$ ) hat dasselbe Verfahren für den Nachweis benutzt. C. Neuberg ${ }^{2}$ ) hat an die Stelle von Quecksilbernitrat Quecksilberacetat gesetzt und zwar in der Form, daß man den Harn mit Quecksilberacetat verreibt. Auch für die qualitative Prüfung ist dieses Verfahren schon von Neuberg ${ }^{3}$ ) empfohlen worden, das überschüssige Quecksiiber muß zur Anstellung der Reaktion durch Zinkstaub entfernt werden. Ich führe diese Probe unter möglichster Vermeidung eines Überschusses von Quecksilberacetat in folgender Weise aus.

Man versetzt ca. $20 \mathrm{ccm}$ des Harns im Bechergläschen so lange mit $20 \%$ iger oder auch noch konzentrierterer Quecksilberacetatlösung, als noch ein deutlicher Niederschlag entsteht, setzt alsdann Natriumcarbonatlösung bis zur neutralen oder bleibenden ganz schwach sauren Reaktion hinzu (nicht zur alkalischen!) filtriert nach einigen Minuten ca. $10 \mathrm{ccm}$ ab. Das Filtrat säuert man im Reagenzglas mit einem bis einigen Tropfen Salzsäure an, fügt eine starke Messerspitze Zinkpulver hinzu (Zink, gepulvert, von Kahlbau m ist dem technischen Zinkstaub vorzuziehen), erwärmt gelind und schüttelt einige Male durch, indem man das Reagenzglas mit dem Daunen verschließt und

1) Spaeth, Untersuchung des Harns, Leipzig 1908, S. 268.

2) Biochem. Zeitschr., Bd. 24, S. 424 (1910).

s) „Der Harn` usw., Handbuch, Berlin 1911, S. 333. 
nach jedesmaligem Schütteln den Verschluß lüftet. Man filtriert, überzeugt sich an einer kleinen abgenommenen Probe des Filtrates, ob dasselbe quecksilberfrei ist, ${ }^{1}$ ) und stellt dann mit dem Filtrat in der gewöhnlichen Weise die Trommersche Probe an unter Anwendung des halben Volumens Natronlauge von $15 \%$ und einem kleinen Überschuß von $10 \%$ iger Kupfersulfatlösung. Handelt es sich um normalen Harn, so behält diè Flüssigkeit beim Erhitzen ihre blaue Farbe, hat man dem Harn $0,2 \%$ Traubenzucker hinzugesetzt, so wird sie intensiv gelb, in der Regel scheidet sich auch nach einer halben bis ganzen Minute gelbes Kupferoxydulhydrat aus. Auch hier ist die Abscheidung durch Salzsäurezusatz zu befördern. Die Probe nimmt nicht viel Zeit fort und scheint mir recht sicher und bequem, jedenfalls möchte ich sie zur weiteren Prüfung empfehlen.

Beiläufig möchte ich noch bemerken, daß ich in Übereinstimmung mit Hammarsten ${ }^{2}$ ) die Almén-Nylandersche Probe nicht so unsicher finde, wie Pflüger ${ }^{3}$ ) sie seinerzeit hingestellt hat. Es kommt ja wohl vor, daß sie positiv ausfällt trotz Fehlens von Zucker und ohne daß man den Gebrauch von Medikamenten dafür verantwortlich machen kann, aber doch selten und nur bei sehr konzentriertem Harn.

1) Am einfachsten durch Zusatz von Salzsäure und Zinnchlorürlösung. - Bei unzureichendem Salzsäurezusatz entsteht ein weißer Niederschlag (phosphorsaures Zinn?).

2) Pflügers Archiv, Bd. 116, S. 517.

3) Pflügers Archiv, Bd. 116, S. 265 u. 533. 